\title{
Prediction of survival in patients with IDH-wildtype astrocytic gliomas using dynamic $\mathrm{O}-\left(2-\left[{ }^{18} \mathrm{~F}\right]\right.$-fluoroethyl)-L-tyrosine PET
}

\author{
Elena K. Bauer ${ }^{1}$ - Gabriele Stoffels ${ }^{2}$ - Tobias Blau ${ }^{3,4}$ • Guido Reifenberger ${ }^{5,6}$ • Jörg Felsberg ${ }^{5,6}$ • Jan M. Werner ${ }^{1}$. \\ Philipp Lohmann ${ }^{2}$ - Jurij Rosen ${ }^{1} \cdot$ Garry Ceccon ${ }^{1}$ - Caroline Tscherpel ${ }^{1}$ - Marion Rapp ${ }^{6,7}$. Michael Sabel ${ }^{6,7}$. \\ Christian P. Filss ${ }^{2,8} \cdot$ Nadim J. Shah $^{2,9} \cdot$ Bernd Neumaier $^{2} \cdot$ Gereon R. Fink $^{1,2} \cdot$ Karl-Josef Langen $^{2,8,10}$ • \\ Norbert Galldiks ${ }^{1,2,11}$ (D)
}

Received: 7 October 2019 / Accepted: 12 January 2020 / Published online: 7 February 2020

(C) The Author(s) 2020

\begin{abstract}
Purpose Integrated histomolecular diagnostics of gliomas according to the World Health Organization (WHO) classification of 2016 has refined diagnostic accuracy and prediction of prognosis. This study aimed at exploring the prognostic value of dynamic $O-\left(2-\left[{ }^{18} \mathrm{~F}\right]-\right.$ fluoroethyl)-L-tyrosine (FET) PET in newly diagnosed, histomolecularly classified astrocytic gliomas of WHO grades III or IV.

Methods Before initiation of treatment, dynamic FET PET imaging was performed in patients with newly diagnosed glioblastoma $(\mathrm{GBM})$ and anaplastic astrocytoma (AA). Static FET PET parameters such as maximum and mean tumour/brain ratios $\left(\mathrm{TBR}_{\max / \text { mean }}\right)$, the metabolic tumour volume (MTV) as well as the dynamic FET PET parameters time-to-peak (TTP) and slope, were obtained. The predictive ability of FET PET parameters was evaluated concerning the progression-free and overall survival (PFS, OS). Using ROC analyses, threshold values for FET PET parameters were obtained. Subsequently, univariate Kaplan-Meier and multivariate Cox regression survival analyses were performed to assess the predictive power of these parameters for survival.

Results Sixty patients (45 GBM and 15 AA patients) of two university centres were retrospectively identified. Patients with isocitrate dehydrogenase (IDH)-mutant or $\mathrm{O}^{6}$-methylguanine-DNA-methyltransferase (MGMT) promoter-methylated tumours had a significantly longer PFS and OS (both $P<0.001)$. Furthermore, ROC analysis of IDH-wildtype glioma patients $(n=45)$ revealed that a TTP $>25$ min (AUC, 0.90; sensitivity, 90\%; specificity, 87\%; $P<0.001$ ) was highly prognostic for longer PFS (13 vs. 7 months; $P=$ $0.005)$ and $\mathrm{OS}(29$ vs. 12 months; $P<0.001)$. In contrast, at a lower level of significance, $\mathrm{TBR}_{\max }, \mathrm{TBR}_{\text {mean }}$, and MTV were only prognostic for longer OS ( $P=0.004, P=0.038$, and $P=0.048$, respectively). Besides complete resection and a methylated MGMT promoter, TTP remained significant in multivariate survival analysis (all $P \leq 0.02$ ), indicating an independent predictor for OS.

Conclusions Our data suggest that dynamic FET PET allows the identification of patients with longer OS among patients with newly diagnosed IDH-wildtype GBM and AA.
\end{abstract}

Keywords FET PET $\cdot$ High-grade glioma $\cdot$ IDH mutation $\cdot$ MGMT promoter methylation · Overall survival

This article is part of the Topical Collection on Oncology - Brain

Norbert Galldiks

n.galldiks@fz-juelich.de; norbert.galldiks@uk-koeln.de

1 Department of Neurology, Faculty of Medicine and University Hospital Cologne, University of Cologne, Kerpener St. 62, 50937 Cologne, Germany

2 Institute of Neuroscience and Medicine (INM-3, -4, -5), Research Centre Juelich, Leo-Brandt-St. 5, 52425 Juelich, Germany

3 Department of Neuropathology, Faculty of Medicine and University Hospital Cologne, University of Cologne, Cologne, Germany

4 Present address: Institute of Neuropathology, University Hospital Essen, Essen, Germany

5 Institute of Neuropathology, Heinrich Heine University, Duesseldorf, Germany
6 Center of Integrated Oncology (CIO), University of Duesseldorf, Duesseldorf, Germany

7 Department of Neurosurgery, Heinrich Heine University, Duesseldorf, Germany

8 Department of Nuclear Medicine, University Hospital RWTH Aachen, Aachen, Germany

9 Department of Neurology, University Hospital RWTH Aachen, Aachen, Germany

10 Center of Integrated Oncology (CIO), University of Aachen, Aachen, Germany

11 Center of Integrated Oncology (CIO), University of Cologne, Cologne, Germany 


\section{Introduction}

For decades, important general clinical prognostic factors in patients with malignant gliomas including glioblastoma have been patient age, the extent of tumour resection, and the patient's overall clinical status as evaluated by the Karnofsky Performance Score (KPS) [1-6]. More recently, several molecular markers have additionally gained pivotal attention regarding prognostication, and have consecutively contributed to the revised classification of the World Health Organization (WHO) Classification of Tumours of the Central Nervous System 2016 [7]. Since this revision, the classification integrates histologic features and selected molecular biomarkers, in particular the mutation status of the isocitrate dehydrogenase (IDH) genes 1 or 2 and the $1 \mathrm{p} / 19 \mathrm{q}$ co-deletion status. Compared with the previous WHO classification of 2007 that was based on histological features only [8], the inclusion of these biomarkers markedly improved diagnostic accuracy and allowed for a better prediction of the individual prognosis $[9$, 10]. Importantly, the prognosis of most patients with IDHwildtype astrocytic gliomas corresponding histologically to WHO grades II or III has been found to be comparable to that of patients with glioblastoma (GBM) of WHO grade IV, suggesting that genotype is prognostically more important than phenotype in these most common group of gliomas [10,11].

Besides the estimation of patients' prognosis using clinical or molecular parameters, imaging parameters obtained from amino acid PET seems to be of value for prognostic estimations in newly diagnosed and untreated glioma patients. As suggested by the response assessment in neuro-oncology (RANO) working group [12], amino acid PET can provide relevant additional information in clinically equivocal situations, and, moreover, prognostic information of glioma patients prior to treatment, which may be of great value for patient counselling.

The increased uptake of amino acids such as $\mathrm{O}-\left(2-\left[{ }^{18} \mathrm{~F}\right]\right.$ fluoroethyl)-L-tyrosine (FET) by glioma compared with healthy brain parenchyma seems to be caused predominantly by increased transport of large neutral amino acids through the plasma membrane via the L-type amino acid transporter (LAT) system, especially by the subtypes LAT1 and LAT2 [13]. Besides static PET imaging using radiolabelled amino acids [12,14], the value of dynamic PET imaging using the tracer FET has gained attraction for glioma grading [15, 16], the detection of the most malignant foci within nonenhancing gliomas on MRI $[17,18]$, the diagnosis of treatment-related changes such as radiation injury or pseudoprogression [19-23], and the assessment of prognosis in newly diagnosed and untreated gliomas. Regarding the latter indication, the value of dynamic FET PET for prognostication has been demonstrated for gliomas characterized by the 2007 WHO classification $[24,25]$ as well as by the WHO classification of 2016 [18, 26, 27].
In addition to the prognostic importance of the IDHmutation status for glioma patients, recent PET studies suggest that within patients with molecularly defined gliomas the further identification of patients with an additional survival benefit using dynamic FET PET parameters is possible [18, 26, 27]. Over and above the prognostic benefit of an IDH mutation, the prediction of an additional survival benefit derived from dynamic FET PET parameters has been predominantly observed for patients with IDH-mutant astrocytic gliomas of the WHO grades II and III [18, 26, 27].

To date, studies evaluating the prognostic value of dynamic FET PET in prognostically unfavourable gliomas defined by neuropathological characteristics remain scarce. Thus, we evaluated in a selected patient subgroup with newly diagnosed and prognostically unfavourable IDH-wildtype astrocytic gliomas of WHO grades III and IV the value of static and dynamic FET PET parameters regarding their significance to predict prognosis before treatment initiation.

\section{Methods}

\section{Patients}

We retrospectively assessed data from 60 adult glioma patients (median age, 55 years; age range, $21-78$ years; 25 women and 35 men) of two university centres with neuropathologically confirmed newly diagnosed malignant astrocytic glioma (GBM, $n=45$; anaplastic astrocytoma (AA), $n=15$ ) who underwent dynamic FET PET imaging at the Research Centre Juelich, Germany, at initial diagnosis, i.e. before biopsy, resection, or any other kind of treatment. The data had been acquired from 2009 to 2017. Clinical data of all patients, including first-line treatment, are listed in Table 1. The local ethics committees approved the retrospective analysis of the data. There was no conflict with the Declaration of Helsinki. Before PET imaging, all patients had given written informed consent for the PET investigation and the use of the data for scientific purposes.

\section{FET PET imaging}

As described previously, the amino acid FET was produced via nucleophilic ${ }^{18} \mathrm{~F}$-fluorination with a radiochemical purity of greater than $98 \%$, specific radioactivity greater than $200 \mathrm{GBq} / \mu \mathrm{mol}$, and a radiochemical yield of about $60 \%$ [28]. According to national and international guidelines for brain tumour imaging using labelled amino acid analogues $[29,30]$, all patients fasted for at least $4 \mathrm{~h}$ before the PET measurements. All patients underwent a dynamic PET scan from 0 to $50 \mathrm{~min}$ post-injection of $3 \mathrm{MBq}$ of FET per $\mathrm{kg}$ of body weight. PET imaging was performed either on an ECAT Exact HR+PET scanner ( $n=37$ patients) in 3-dimensional 
Table 1 Patient characteristics

\begin{tabular}{ll}
\hline Characteristic & Number \\
\hline Patients & 60 \\
Gender (m/f) & $35 / 25$ \\
Median age; range & $55 ; 21-78$ years \\
Median Karnofsky Performance Score; range & $100 \% ; 60-100 \%$ \\
WHO grade III anaplastic astrocytoma & 15 \\
IDH mutant & 8 \\
IDH wildtype & 7 \\
MGMT promoter methylated & $12(80 \%)$ \\
Contrast enhancement & $10(67 \%)$ \\
Positive FET uptake* & $12(80 \%)$ \\
WHO grade IV glioblastoma & 45 \\
IDH mutant & 7 \\
IDH wildtype & 38 \\
MGMT promoter methylated & $23(51 \%)$ \\
Contrast enhancement & $44(98 \%)$ \\
Positive FET uptake* & $44(98 \%)$ \\
Biopsy & 21 \\
Partial resection & 10 \\
Complete resection & 29 \\
First-line therapy following resection/biopsy & \\
Chemoradiation with temozolomide ${ }^{1}$ & $49(81 \%)$ \\
RT + lomustine-temozolomide ${ }^{2}$ & $2(4 \%)$ \\
RT + bevacizumab ${ }^{3}$ & $1(2 \%)$ \\
Radiotherapy alone & $8(13 \%)$ \\
\hline & \\
\hline
\end{tabular}

$f$ female, IDH isocitrate dehydrogenase, $m$ male, $M G M T \mathrm{O}^{6}$ methylguanine-DNA-methyltransferase, $R T$ radiotherapy,

*FET uptake higher than the background activity, visually assessed by two experienced raters

${ }^{1}$ Treatment according to Stupp et al. [40]

${ }^{2}$ Treatment according to Herrlinger et al. [45]

${ }^{3}$ Treatment according to Herrlinger et al. [46]

mode (Siemens, Erlangen, Germany) (axial field-of-view, $15.5 \mathrm{~cm}$ ) or simultaneously with $3 \mathrm{~T}$ MR imaging using a BrainPET insert ( $n=23$ patients) (Siemens, Erlangen, Germany). The BrainPET is a compact cylinder that fits into the bore of the Magnetom Trio MR scanner (axial field of view, $19.2 \mathrm{~cm})$ [31].

Iterative reconstruction parameters were 16 subsets, 6 iterations using the OSEM algorithm for the ECAT HR+ PET scanner and two subsets, and 32 iterations using the OP-OSEM algorithm for the BrainPET. Data were corrected for random, scattered coincidences, dead time, and motion for both systems. Attenuation correction for the ECAT HR+PET scan was based on a transmission scan, and for the BrainPET scan, a template-based approach [31]. The reconstructed dynamic data sets consisted of 16 time frames $(5 \times 1 \mathrm{~min} ; 5 \times 3 \mathrm{~min} ; 6 \times 5 \mathrm{~min})$ for both scanners.
To optimize comparability of the results related to the influence of the two different PET scanners, reconstruction parameters, and post-processing steps, a $2.5-\mathrm{mm} 3 \mathrm{D}$ Gaussian filter was applied to the BrainPET data before further processing, resulting in an image resolution of approximately $4 \mathrm{~mm}$ full width at half maximum (FWHM) (image resolution of the ECAT HR+PET scanner, approximately 6-mm FWHM). In phantom experiments using spheres of different sizes to simulate lesions, this filter kernel demonstrated the best comparability between PET data obtained from the ECAT HR+PET and the BrainPET scanner [32].

\section{FET PET data analysis}

FET PET data analysis was performed as described previously [33]. In brief, for the evaluation of FET data, summed PET images over 20-40 min post-injection were used. A larger crescent shaped reference ROI was placed in the semioval centre of the contralateral unaffected hemisphere including white and grey matter [30]. Mean amino acid uptake in the tumour was determined by a 2-dimensional auto-contouring process in the transversal slice containing the voxel with the maximum tumour uptake using a tumour-to-brain ratio (TBR) of 1.6 , as described previously $[34,35]$. For the calculation of the maximal amino acid uptake, a circular region-of-interest (ROI) with a diameter of $1.6 \mathrm{~cm}$ was centred on the voxel with the maximum tumour uptake [33]. Maximum and mean TBRs $\left(\mathrm{TBR}_{\max }, \mathrm{TBR}_{\text {mean }}\right)$ were calculated by dividing the mean SUV of the tumour ROIs by the mean SUV of healthy brain tissue. The FET metabolic tumour volume (MTV) was determined by a 3-dimensional auto-contouring process using a TBR of 1.6 or more using the software PMOD (Version 3.9, PMOD Technologies Ltd.).

As described previously [33], time-activity curves (TACs) of FET uptake (mean SUV) in the tumour were generated by the application of a spherical volume-of-interest (VOI) with a volume of $2 \mathrm{~mL}$ centred on the voxel with the maximum tumour uptake and the reference ROI as described above to the entire dynamic dataset. A reference TAC was generated by placing a reference ROI in the unaffected brain tissue as reported [33]. For TAC evaluation, the time-to-peak (TTP; time in minutes from the beginning of the dynamic acquisition up to the maximum SUV of the lesion) was determined. In cases with steadily increasing FET uptake without identifiable peak uptake, we defined the end of the dynamic PET acquisition as TTP. Furthermore, the slope of the TAC in the late phase of FET uptake was assessed by fitting a linear regression line to the late phase of the curve (20-50 min post-injection). The slope was expressed as the change of the SUV per hour. This procedure allows for a more objective evaluation of kinetic data compared with an assignment of TACs to earlier reported patterns of FET uptake during dynamic acquisition [33]. 
The ROIs and VOIs are selected, positioned and validated by two experienced, board-certified specialists in nuclear medicine (G.S., K-J.L.) with more than 10 years of experience in the analysis of FET PET images.

\section{Neuropathological tumour classification and analysis of molecular markers}

All tumours were neuropathologically re-classified according to the WHO Classification of Tumours of the Central Nervous System of 2016 [7]. For molecular biomarker analysis, tumour DNA was extracted from formalin-fixed and paraffinembedded tissue samples with a histologically estimated tumour cell content of $80 \%$ or more. For assessment of the IDH mutation status, the presence of an IDH1-R132H mutation was evaluated by immunohistochemistry using a mutationspecific antibody in a standard immunohistochemical staining procedure as reported before [36, 37]. When immunostaining for IDH1-R132H remained negative, the mutational hot-spots at codon 132 of IDH1 and codon 172 of IDH2 were directly sequenced as reported $[10,38]$. The $1 \mathrm{p} / 19 \mathrm{q}$ co-deletion status was determined by PCR-based microsatellite analysis as reported [39]. The $\mathrm{O}^{6}$-methylguanine-DNA-methyltransferase (MGMT) promoter methylation status was assessed by methylation-specific PCR as described elsewhere [38].

\section{Survival times}

Progression-free survival (PFS) was defined as the time in months between the neuropathologically confirmed glioma diagnosis and tumour progression according to the response assessment in neuro-oncology (RANO) working group [40]. The overall survival (OS) was defined as the time in months between the neuropathologically confirmed glioma diagnosis and death.

\section{Statistical analyses}

Descriptive statistics are provided as mean and standard deviation or median and range. The Student $t$ test for independent samples was used to compare two different groups. The Mann-Whitney rank-sum test was used when variables were not normally distributed. The diagnostic performance of FET uptake, as determined by $\mathrm{TBR}_{\max }$, $\mathrm{TBR}_{\text {mean }}$, TTP, and slope, was assessed by receiver operating characteristic (ROC) curve analyses using a favourable OS of $\geq 24$ months as reference (the median OS in GBM patients is in the range of 1520 months [41-43], and the 2-year survival rate is $30 \%$ [44]; therefore, an $\mathrm{OS}$ of $\geq 24$ months was considered as favourable). The decision cutoff was considered optimal when the product of paired values for sensitivity and specificity reached its maximum. As a measure of the diagnostic quality of the test, we determined the area under the ROC curve
(AUC), its standard error, and the level of significance. Univariate survival analyses were performed using the logrank test. Multivariate Cox proportional hazards models were constructed to test the relationship between static and dynamic FET PET parameters and other predictors of survival. Parameters that were significant in univariate analyses were included in multivariate models. A $P$ value of $<0.05$ was considered significant. Statistical analyses were performed using SigmaStat software (SigmaPlot for Windows 11.0, Chicago, IL) and SPSS Statistics software (Release 24.0, SPSS Inc., Chicago, IL, USA).

\section{Results}

\section{Survival}

In the whole cohort $(n=60)$, the median PFS was 12 months (range, 0-75 months), and the median OS was 17 months (range, 0-75 months). In the subgroup of patients with IDHwildtype gliomas $(n=45)$, the median PFS was 9 months (range, 0-52 months), and the median OS was 15 months (range, 0-52 months). Following biopsy or resection, the vast majority of patients underwent radiotherapy plus an adjuvant treatment option $(n=52 ; 87 \%)$ (Table 1$)$.

\section{Optimal thresholds derived from FET PET parameters}

An overview of the results of the optimal thresholds derived from FET PET is provided in Table 2. Specifically, ROC analysis revealed that the dynamic FET PET parameter slope predicts a favourable OS of $\geq 24$ months with a sensitivity of $70 \%$ and a specificity of $90 \%$ (AUC, $0.77 \pm 0.09$; threshold, $0.103 \mathrm{SUV} / \mathrm{h} ; P=0.010$ ). The most significant $\mathrm{OS}$ prediction of $\geq 24$ months could be obtained by the dynamic FET PET parameter TTP (threshold, 25 min; sensitivity 90\%; specificity, 87\%; AUC, $0.90 \pm 0.07 ; P<0.001$ ) (Figs. 1, 2, and 3). In contrast, the static FET PET parameters $\mathrm{TBR}_{\max }, \mathrm{TBR}_{\text {mean }}$, and MTV were not prognostic for a favourable OS of $\geq$ 24 months (AUC, 0.63, 0.69, and 0.56, respectively).

\section{Univariate survival analysis-influence of general prognostic factors on survival}

In the whole cohort $(n=60)$, the univariate survival analysis revealed that glioma patients with IDH-mutant tumours had significantly longer PFS (33 vs. 9 months) and OS (54 vs. 13 months) than patients with IDH-wildtype gliomas (both $P<0.001)$. Similarly, glioma patients with MGMT promoter-methylated tumours had a significantly longer PFS (18 vs. 7 months) and OS (29 vs. 12 months) than glioma patients whose tumours lacked MGMT promoter methylation (both $P<0.001$ ). Patients with a KPS of $100 \%$ had a 
Table 2 Diagnostic performance of static and dynamic FET PET parameters

\begin{tabular}{llllll}
\hline & TBR $_{\text {max }}$ & TBR $_{\text {mean }}$ & MTV & TTP & Slope \\
\hline Threshold & 2.55 & 2.05 & $11.15 \mathrm{~mL}$ & $25 \mathrm{~min}$ & $-0.103 \mathrm{SUV} / \mathrm{h}$ \\
Sensitivity (\%) & 70 & 60 & $70 \%$ & 90 & 70 \\
Specificity (\%) & 57 & $0.69 \pm 0.09$ & $0.56 \pm 0.09$ & $0.90 \pm 0.07$ & $0.77 \pm 0.09$ \\
AUC \pm standard deviation & $0.63 \pm 0.10$ & 0.083 & 0.56 & $<0.001$ & 0.010 \\
$P$ value & 0.235 & & & $54 \%$ & 80 \\
\hline
\end{tabular}

$A U C$ area under curve from a receiver operating characteristic curve, $M T V$ metabolic tumour volume, $T B R_{\max }$ maximum tumour-to-brain ratio of FET uptake, $T B R_{\text {mean }}$ mean tumour-to-brain ratio of FET uptake, TTP time-to-peak; SUV standardized uptake value

significantly longer OS than patients with a KPS between 60 and $90 \%$ (29 vs. 13 months; $P=0.015$ ). Furthermore, patients with complete resection of the glioma at initial diagnosis had a significantly longer PFS compared with incompletely resected patients or patients who received a biopsy only (13 vs. 10 months; $P=0.038)$, but not a significantly longer OS $(P=0.121)$. Patients aged $<70$ years at initial diagnosis did not show longer PFS and OS compared with patients $\geq$ 70 years $(P=0.552$ and $P=0.108$, respectively) (Table 3$)$.

\section{Univariate survival analysis-prediction of survival using static and dynamic FET PET parameters}

In patients diagnosed with an IDH-wildtype glioma at initial diagnosis $(n=45)$, the dynamic FET PET parameter TTP (threshold, $25 \mathrm{~min}$ ) predicted both a significantly longer PFS (13 vs. 7 months) and OS (29 vs. 12 months) $(P=0.005$ and $P<0.001$, respectively) (Figs. 4 and 5). Furthermore, the dynamic FET PET parameter slope (threshold, $-0.103 \mathrm{SUV} / \mathrm{h}$ ) also predicted a significantly longer OS (17 vs. 9 months; $P=$ $0.021)$, but not a significantly longer PFS $(P=0.065)$.

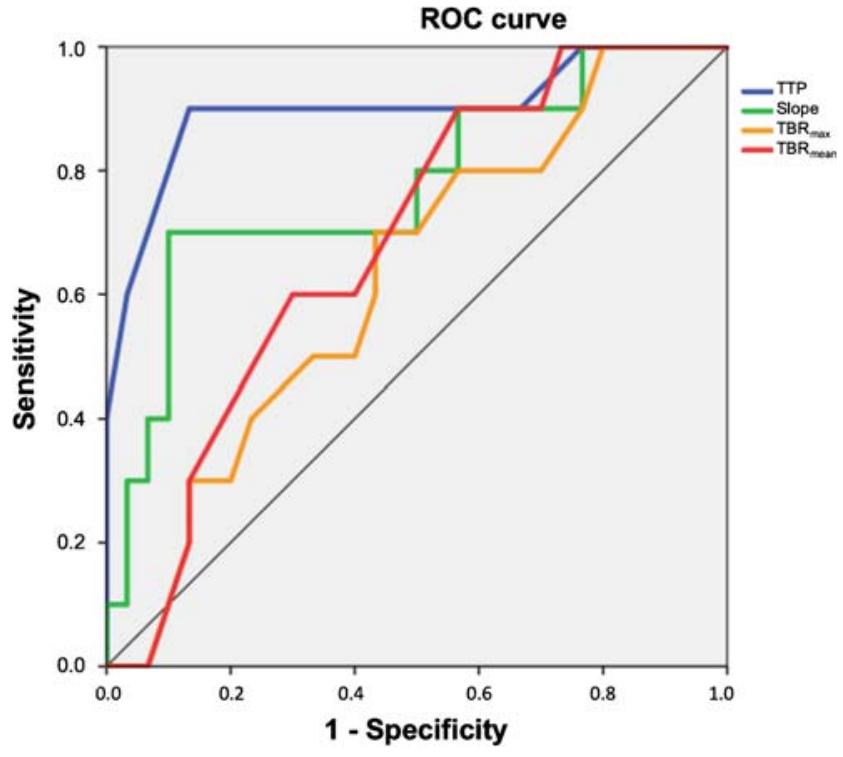

Fig. 1 ROC curves for the parameters TTP, slope, $\mathrm{TBR}_{\max }$, and $\mathrm{TBR}_{\text {mean }}$
Compared with TTP, $\mathrm{TBR}_{\max }$, $\mathrm{TBR}_{\text {mean }}$, and MTV were only prognostic for a longer $\mathrm{OS}$ at a lower level of significance ( $P=0.004, P=0.038$, and $P=0.048$, respectively) (Table 3 ).

\section{Multivariate survival analysis}

Besides MGMT promoter methylation and complete resection at initial diagnosis, the dynamic FET PET parameter TTP remained statistically significant in the multivariate survival analysis $(P=0.008$; HR, 0.941; 95\% CI, 0.900-0.984), indicating an independent predictor for OS. The parameters KPS, $\mathrm{TBR}_{\text {max }}, \mathrm{TBR}_{\text {mean }}$, slope, and MTV were not statistically significant in the multivariate survival analysis (Table 4).

\section{Discussion}

The main finding of the present study is that dynamic FET PET parameters such as TTP and slope may identify a prognostically more favourable subgroup among patients with newly diagnosed IDH-wildtype astrocytic glioma of the WHO grades III or IV. Our findings suggest that imaging biomarkers derived from dynamic FET PET provide additional prognostic information beyond molecular biomarkers and WHO grades. Thus, FET PET may be valuable for patient counselling and inform treatment planning, thereby allowing stronger emphasis on personalized therapies based on both molecular markers and refined imaging techniques such as dynamic FET PET.

Basically, our findings are line with and extent previous results [18, 26, 27]. Studies in patients with lower-grade and mainly non-enhancing IDH-mutant astrocytomas also highlighted the value of dynamic FET PET parameters (i.e. TAC patterns and TTP) to independently identify patient subgroups with favourable outcome regarding PFS and OS. In comparison to the present study, however, there are differences which warrant discussion.

Like in the present study, Suchorska and colleagues [27] reported in IDH-mutant gliomas of WHO grade II or III an optimal TTP threshold of $>25$ min to identify patients with favourable outcome. Using a more stringent TTP threshold of $>12.5 \mathrm{~min}$, the identification of IDH-wildtype glioma patients 
Fig. 2 Neuroimages including FET PET, contrast-enhanced MRI, FLAIR-weighted MR image, and the TAC of a patient with an IDH-wildtype GBM and prognostically unfavourable dynamic FET PET parameters (i.e. TTP $<25$ min, slope $<-0.103$ $\mathrm{SUV} / \mathrm{h})$. The OS of that patient was 14 months
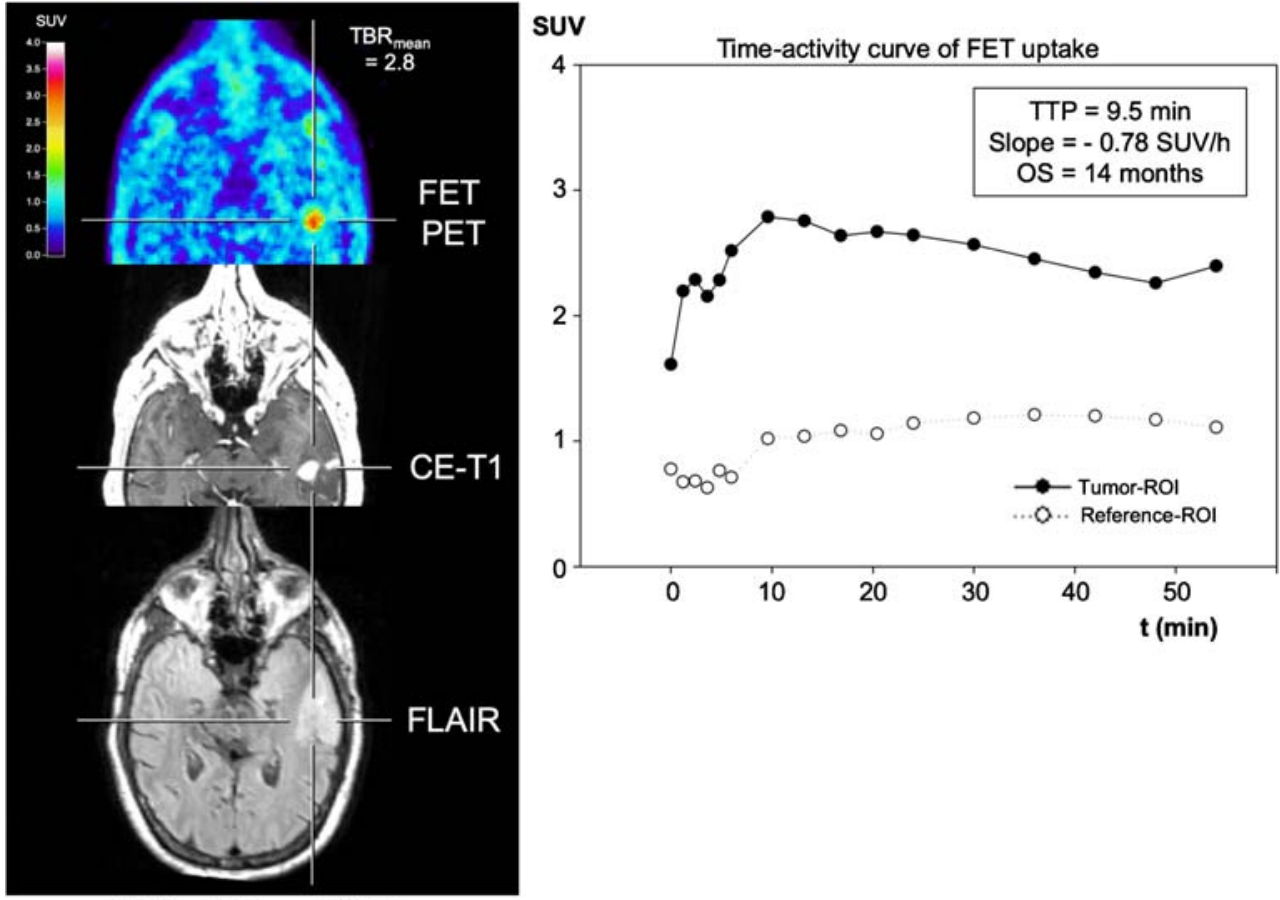

of WHO grade II or III $(n=76)$ with favourable outcome was, however, not possible. Conversely, a recent study reported that the TTP threshold of $12.5 \mathrm{~min}$ allows the distinction between two prognostic subgroups, including lower-grade IDHwildtype glioma patients $(n=27)$ [27]. We here demonstrate that the identification of two distinct prognostic subgroups among patients with IDH-wildtype astrocytic gliomas of WHO grades III or IV is possible using a TTP threshold of $25 \mathrm{~min}$. As suggested previously [45], one reason might be the slightly different dynamic FET PET imaging protocol used in different centres, e.g. scanning time of $50 \mathrm{~min}$ in our centre compared with $40 \mathrm{~min}$ in other centres [18, 26, 27].
Fig. 3 Neuroimages including FET PET, contrast-enhanced MRI, FLAIR-weighted MR image, and the TAC of a patient with an IDH-wildtype GBM and prognostically favourable dynamic FET PET parameters (i.e. TTP $>25$ min, slope $>-0.103$ $\mathrm{SUV} / \mathrm{h}$ ). The OS of that patient was 30 months
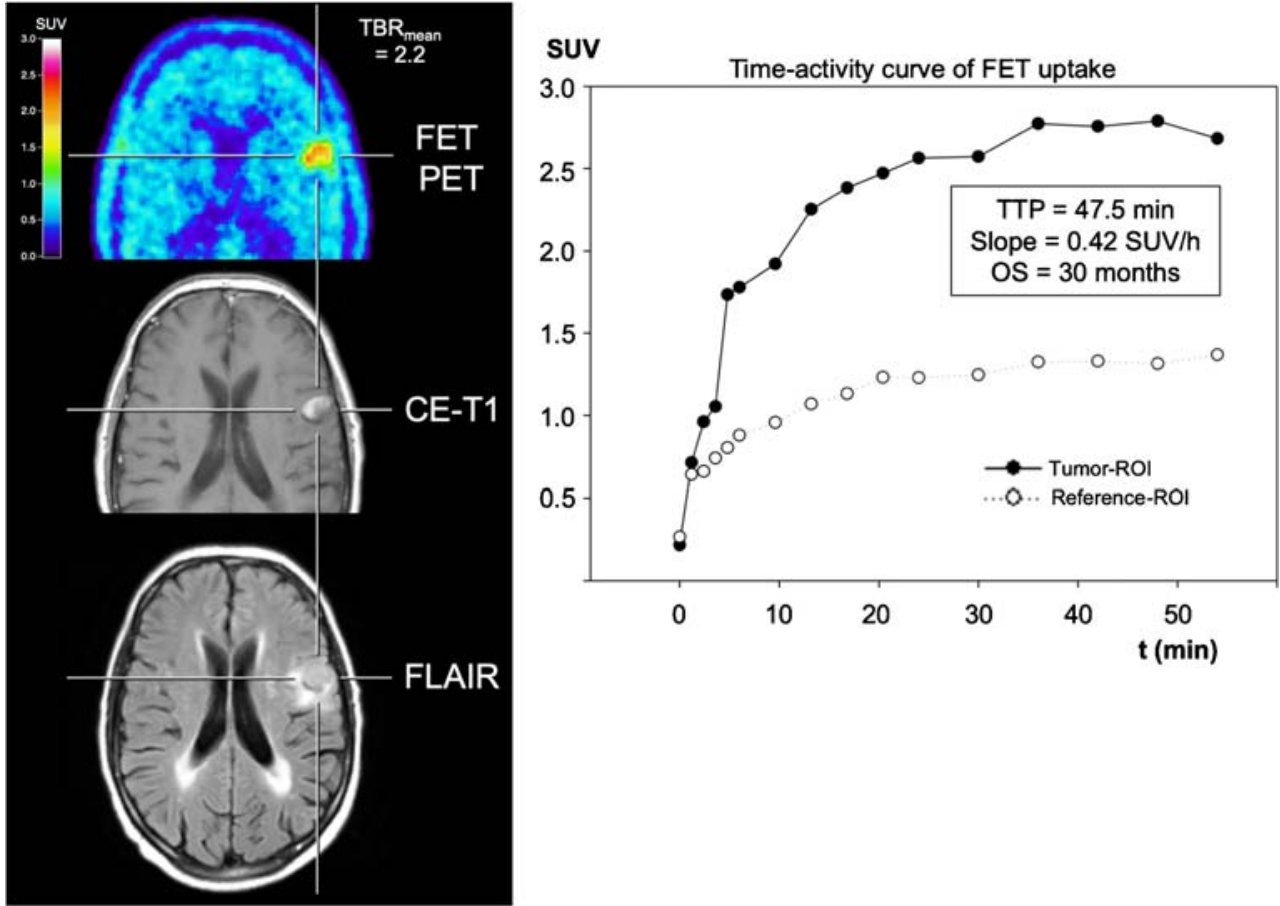

IDH-wildtype GBM 
Table 3 Results of univariate survival analyses

\begin{tabular}{|c|c|c|c|c|c|}
\hline \multirow[t]{2}{*}{ Factor } & \multirow[t]{2}{*}{ Threshold/criterion } & \multicolumn{2}{|l|}{ PFS } & \multicolumn{2}{|l|}{ OS } \\
\hline & & $P$ value & Survival time & $P$ value & Survival time \\
\hline Age & $<70$ vs. $>70$ years & 0.552 & 12 vs. 9 months & 0.108 & 22 vs. 17 months \\
\hline Resection & CR vs. B/PR & 0.038 & 13 vs. 10 months & 0.121 & 23 vs. 15 months \\
\hline IDH mutation & mutant vs. wildtype & $<0.001$ & 33 vs. 9 months & $<0.001$ & 54 vs. 13 months \\
\hline MGMT promoter & methylated vs. non-methylated & $<0.001$ & 18 vs. 7 months & $<0.001$ & 29 vs. 12 months \\
\hline KPS & $100 \%$ vs. $<100 \%$ & 0.143 & 14 vs. 9 months & 0.015 & 29 vs. 13 months \\
\hline $\mathrm{TBR}_{\max }$ & $<2.55$ vs. $>2.55$ & 0.072 & 12 vs. 7 months & 0.004 & 24 vs. 12 months \\
\hline $\mathrm{TBR}_{\text {mean }}$ & $<2.05$ vs. $>2.05$ & 0.112 & 14 vs. 7 months & 0.038 & 25 vs. 12 months \\
\hline TTP & $<25$ vs. $>25 \mathrm{~min}$ & 0.005 & 13 vs. 7 months & $<0.001$ & 29 vs. 12 months \\
\hline Slope & $>-0.103$ vs. $<-0.103 \mathrm{SUV} / \mathrm{h}$ & 0.065 & 9 vs. 6 months & 0.021 & 17 vs. 9 months \\
\hline MTV & $11.15 \mathrm{~mL}$ & 0.406 & 12 vs. 7 months & 0.048 & 24 vs. 12 months \\
\hline
\end{tabular}

$B$ biopsy, $C R$ complete resection, $I D H$ isocitrate dehydrogenase, KPS Karnofsky Performance Score, $M G M T$, $\mathrm{O}^{6}$-methylguanine-DNA-methyltransferase, $M T V$, metabolic tumour volume, $O S$ overall survival, $P F S$ progression-free survival, $P R$ partial resection, $T B R_{\max }$ maximum tumour-to-brain ratio of FET uptake, $T B R_{\text {mean }}$ mean tumour-to-brain ratio of FET uptake, $S U V$ standardized uptake value

Furthermore, differences in WHO grades (i.e. a higher proportion of IDH-wildtype gliomas of WHO grade III or IV in the present study) may impact on the TTP threshold.

As reported for several other indications [15-22], TAC patterns (e.g. increasing/decreasing TACs), either combined with TTP [18] or alone [26], also seem to have prognostic significance for glioma subgroups. Notwithstanding, the interpretation of TAC patterns is subjective to a certain degree. To provide a more objective and readerindependent characterization of TAC patterns in the present study, we calculated the slope by fitting a linear regression line to the late phase of the TAC. Importantly, similar to TTP, albeit at a lower level of significance, the slope value also allows identifying patients with an improved OS within neuropathologically defined glioma subgroups.

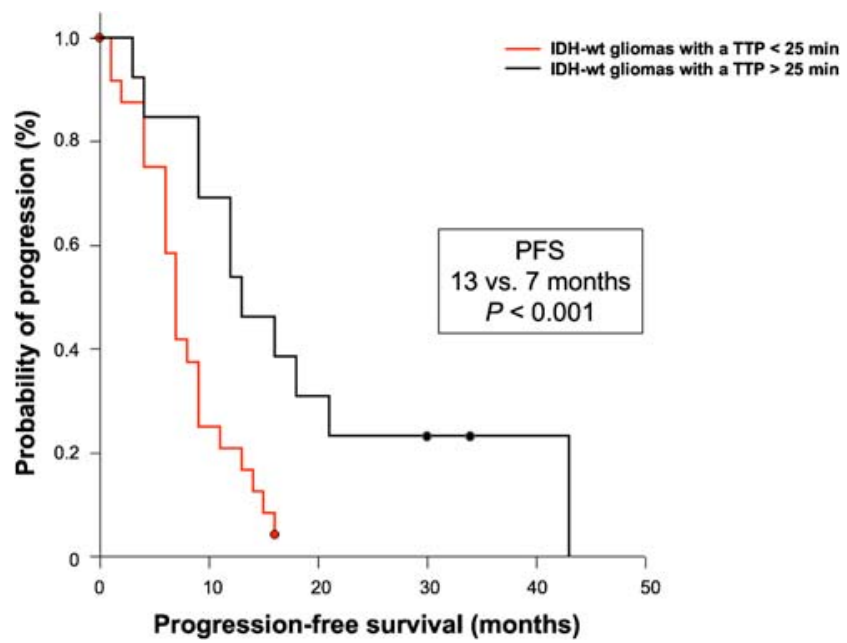

Fig. 4 PFS separated by the TTP (threshold, $25 \mathrm{~min}$ ) within the patient group of newly diagnosed and IDH-wildtype astrocytic glioma of the WHO grades III or IV
The following limitations of our study need to be discussed. The study is based on retrospective data, and the results need to be confirmed in a prospective study. Another putative weakness is the relatively small number of patients. Nevertheless, compared with other studies [18, 26], our dataset includes mostly patients with unfavourable IDHwildtype astrocytic glioma of WHO grades III and IV, allowing a more profound prognostic evaluation within this group of patients. Furthermore, from a practical point of view, the acquisition and post-processing of a dynamic FET PET scan is more complex and time-consuming than the evaluation of TBRs, which are commonly used in clinical routine, and therefore hampers a widespread use of this powerful imaging technique. In addition, one might argue that PET imaging protocols are not sufficiently standardized for widespread

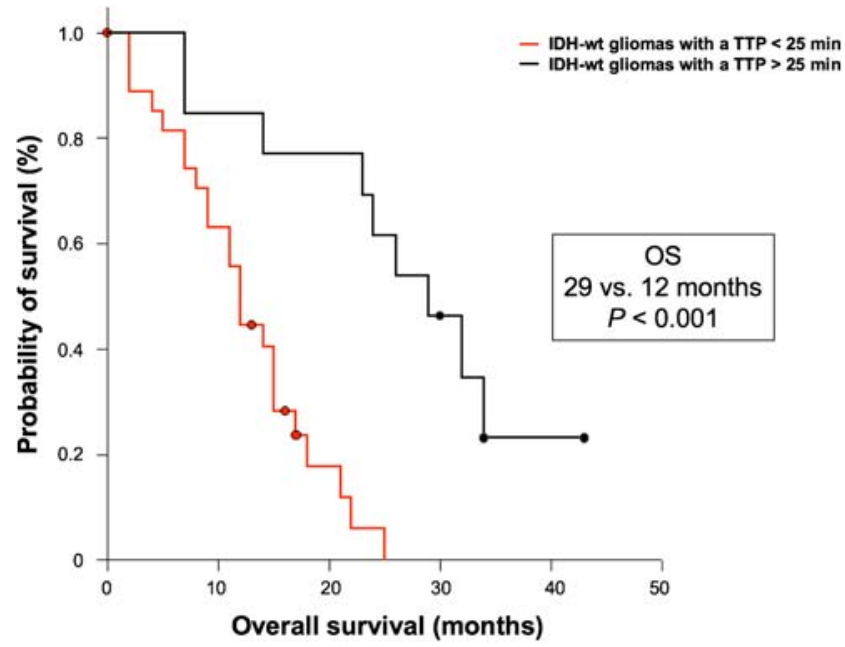

Fig. 5 OS separated by the TTP (threshold, $25 \mathrm{~min}$ ) within the patient group of newly diagnosed and IDH-wildtype astrocytic glioma of the WHO grades III or IV 
Table 4 Results of multivariate survival analyses in patients with IDH-wildtype glioma

\begin{tabular}{lllll}
\hline & Threshold & Hazard ratio & $95 \%$ confidence interval & $P$ value \\
\hline TTP & 25 min & 0.941 & $0.900-0.984$ & 0.008 \\
MGMT promoter & methylated & 0.337 & $0.133-0.851$ & 0.021 \\
KPS & $100 \%$ & 1.680 & $0.752-3.753$ & 0.206 \\
Resection & CR & 0.328 & $0.147-0.730$ & 0.006 \\
TBR $_{\text {max }}$ & 2.55 & 0.844 & $0.127-5.620$ & 0.861 \\
TBR $_{\text {mean }}$ & 2.05 & 0.845 & $0.033-21.852$ & 0.919 \\
Slope & $-0.103 \mathrm{SUV} / \mathrm{h}$ & 1.199 & $0.600-2.395$ & 0.607 \\
MTV & $11.15 \mathrm{~mL}$ & 1.017 & $0.986-1.048$ & 0.294 \\
\hline
\end{tabular}

$C R$ Complete resection, KPS Karnofsky Performance Score, $M G M T \mathrm{O}^{6}$-methylguanine-DNA-methyltransferase, $M T V$ metabolic tumour volume, $T B R_{\max }$ maximum tumour-to-brain ratio of FET uptake, $T B R_{\text {mean }}$ mean tumourto-brain ratio of FET uptake, TTP time-to-peak, $S U V$ standardized uptake value use on a routine basis. Notwithstanding, in 2019, major European and American medical societies for nuclear medicine and neuro-oncology (i.e. the SNMMI (Society of Nuclear Medicine and Molecular Imaging), EANM (European Association of Nuclear Medicine), EANO (European Association of Neuro-Oncology), and the RANO group) have published joint practice guidelines for static and dynamic PET imaging [30] which may help to harmonize acquisition protocols and post-processing in the near future.

Another important point which needs to be discussed is the detection of the most malignant parts for neuropathological evaluation. In the present study, tissue was obtained from tumour parts with the highest metabolic activity on FET PET. It has been demonstrated that this procedure identifies the most malignant tumour parts in heterogeneous gliomas with high accuracy and therefore helps to avoid undergrading [17]. Furthermore, in gliomas with an IDH mutation, mutant IDH proteins are ubiquitously expressed in tumour cells, suggesting that IDH mutations are an early causative event in the genesis of these brain tumours [46]. This also applies to the MGMT methylation status [47], and other molecular markers such as 1p/19q co-deletion and TP53 [48]. Thus, due to a FET PET-guided tissue removal of the tumour parts with the highest metabolic activity and the homogenous distribution of various molecular markers within gliomas, a histomolecular misclassification seems to be unlikely.

In conclusion, our data suggest that within a histomolecularly defined subgroup of patients with newly diagnosed IDH-wildtype GBM and AA, dynamic FET PET allows the identification of patients with improved OS. Especially in the subgroup of patients with IDH-wildtype tumours and reduced clinical condition (e.g. older patients with a reduced KPS), our FET PET findings may be helpful for patient counselling, i.e. to decide whether an aggressive treatment regimen including radio- and chemotherapy with an increased risk of severe side effects should be administered or not. Accordingly, prospective data are warranted to further improve the level of confidence.
Funding Information Open Access funding provided by Projekt DEAL. The Wilhelm-Sander Stiftung, Germany, supported this work.

\section{Compliance with ethical standards}

Conflict of interest The authors declare that they have no conflict of interest.

Ethical approval All procedures performed in studies involving human participants were in accordance with the ethical standards of the institutional and/or national research committee and the 1964 Helsinki declaration and its later amendments or comparable ethical standards.

Informed consent Informed written consent was obtained from all individual participants included in the study.

Open Access This article is licensed under a Creative Commons Attribution 4.0 International License, which permits use, sharing, adaptation, distribution and reproduction in any medium or format, as long as you give appropriate credit to the original author(s) and the source, provide a link to the Creative Commons licence, and indicate if changes were made. The images or other third party material in this article are included in the article's Creative Commons licence, unless indicated otherwise in a credit line to the material. If material is not included in the article's Creative Commons licence and your intended use is not permitted by statutory regulation or exceeds the permitted use, you will need to obtain permission directly from the copyright holder. To view a copy of this licence, visit http://creativecommons.org/licenses/by/4.0/.

\section{References}

1. Curran WJ Jr, Scott CB, Horton J, Nelson JS, Weinstein AS, Fischbach AJ, et al. Recursive partitioning analysis of prognostic factors in three radiation therapy oncology group malignant glioma trials. J Natl Cancer Inst. 1993;85:704-10.

2. Lacroix M, Abi-Said D, Fourney DR, Gokaslan ZL, Shi W, DeMonte F, et al. A multivariate analysis of 416 patients with glioblastoma multiforme: prognosis, extent of resection, and survival. J Neurosurg. 2001;95:190-8.

3. Stummer W, Pichlmeier U, Meinel T, Wiestler OD, Zanella F, Reulen HJ. Fluorescence-guided surgery with 5-aminolevulinic acid for resection of malignant glioma: a randomised controlled multicentre phase III trial. Lancet Oncol. 2006;7:392-401. 
4. Perry JR, Laperriere N, O'Callaghan CJ, Brandes AA, Menten J, Phillips C, et al. Short-course radiation plus temozolomide in elderly patients with glioblastoma. N Engl J Med. 2017;376:1027-37.

5. Wick W, Platten M, Meisner C, Felsberg J, Tabatabai G, Simon M, et al. Temozolomide chemotherapy alone versus radiotherapy alone for malignant astrocytoma in the elderly: the NOA-08 randomised, phase 3 trial. Lancet Oncol. 2012;13:707-15.

6. Kreth FW, Thon N, Simon M, Westphal M, Schackert G, Nikkhah $\mathrm{G}$, et al. Gross total but not incomplete resection of glioblastoma prolongs survival in the era of radiochemotherapy. Ann Oncol. 2013;24:3117-23.

7. Louis DN, Perry A, Reifenberger G, von Deimling A, FigarellaBranger D, Cavenee WK, et al. The 2016 World Health Organization classification of tumors of the central nervous system: a summary. Acta Neuropathol. 2016;131:803-20.

8. Louis DN, Ohgaki H, Wiestler OD, Cavenee WK, Burger PC, Jouvet A, et al. The 2007 WHO classification of tumours of the central nervous system. Acta Neuropathol. 2007;114:97-109.

9. Reuss DE, Mamatjan Y, Schrimpf D, Capper D, Hovestadt V, Kratz A, et al. IDH mutant diffuse and anaplastic astrocytomas have similar age at presentation and little difference in survival: a grading problem for WHO. Acta Neuropathol. 2015;129:867-73.

10. Hartmann C, Hentschel B, Wick W, Capper D, Felsberg J, Simon $\mathrm{M}$, et al. Patients with IDH1 wild type anaplastic astrocytomas exhibit worse prognosis than IDH1-mutated glioblastomas, and IDH1 mutation status accounts for the unfavorable prognostic effect of higher age: implications for classification of gliomas. Acta Neuropathol. 2010;120:707-18.

11. Cancer Genome Atlas Research N, Brat DJ, Verhaak RG, Aldape $\mathrm{KD}$, Yung WK, Salama SR, et al. Comprehensive, integrative genomic analysis of diffuse lower-grade gliomas. N Engl J Med. 2015;372:2481-98.

12. Albert NL, Weller M, Suchorska B, Galldiks N, Soffietti R, Kim $\mathrm{MM}$, et al. Response assessment in neuro-oncology working group and European Association for Neuro-Oncology recommendations for the clinical use of PET imaging in gliomas. Neuro-Oncology. 2016;18:1199-208.

13. Habermeier A, Graf J, Sandhofer BF, Boissel JP, Roesch F, Closs EI. System L amino acid transporter LAT1 accumulates O-(2fluoroethyl)-L-tyrosine (FET). Amino Acids. 2015;47:335-44.

14. Langen KJ, Galldiks N, Hattingen E, Shah NJ. Advances in neurooncology imaging. Nat Rev Neurol. 2017;13:279-89.

15. Pöpperl G, Kreth FW, Herms J, Koch W, Mehrkens JH, Gildehaus FJ, et al. Analysis of 18F-FET PET for grading of recurrent gliomas: is evaluation of uptake kinetics superior to standard methods? J Nucl Med. 2006;47:393-403.

16. Pöpperl G, Kreth FW, Mehrkens JH, Herms J, Seelos K, Koch W, et al. FET PET for the evaluation of untreated gliomas: correlation of FET uptake and uptake kinetics with tumour grading. Eur J Nucl Med Mol Imaging. 2007;34:1933-42.

17. Kunz M, Thon N, Eigenbrod S, Hartmann C, Egensperger R, Herms J, et al. Hot spots in dynamic (18)FET-PET delineate malignant tumor parts within suspected WHO grade II gliomas. Neuro-Oncology. 2011;13:307-16.

18. Kunz M, Albert NL, Unterrainer M, la Fougere C, Egensperger R, Schüller U, et al. Dynamic 18F-FET PET is a powerful imaging biomarker in gadolinium-negative gliomas. Neuro-Oncology. 2019;21:274-84

19. Ceccon G, Lohmann P, Stoffels G, Judov N, Filss CP, Rapp M, et al. Dynamic O-(2-18F-fluoroethyl)-L-tyrosine positron emission tomography differentiates brain metastasis recurrence from radiation injury after radiotherapy. Neuro-Oncology. 2017;19:281-8.

20. Galldiks N, Langen KJ, Albert NL, Chamberlain M, Soffietti R, Kim MM, et al. PET imaging in patients with brain metastasisreport of the RANO/PET group. Neuro-Oncology. 2019;21:58595.
21. Lohmann P, Kocher M, Ceccon G, Bauer EK, Stoffels G, Viswanathan S, et al. Combined FET PET/MRI radiomics differentiates radiation injury from recurrent brain metastasis. Neuroimage Clin. 2018;20:537-42.

22. Galldiks N, Stoffels G, Filss CP, Piroth MD, Sabel M, Ruge MI, et al. Role of O-(2-18F-fluoroethyl)-L-tyrosine PET for differentiation of local recurrent brain metastasis from radiation necrosis. $\mathrm{J}$ Nucl Med. 2012;53:1367-74.

23. Galldiks N, Dunkl V, Stoffels G, Hutterer M, Rapp M, Sabel M, et al. Diagnosis of pseudoprogression in patients with glioblastoma using O-(2-[18F]fluoroethyl)-L-tyrosine PET. Eur J Nucl Med Mol Imaging. 2015;42:685-95.

24. Jansen NL, Suchorska B, Wenter V, Eigenbrod S, SchmidTannwald C, Zwergal A, et al. Dynamic 18F-FET PET in newly diagnosed astrocytic low-grade glioma identifies high-risk patients. J Nucl Med. 2014;55:198-203.

25. Jansen NL, Suchorska B, Wenter V, Schmid-Tannwald C, Todica A, Eigenbrod S, et al. Prognostic significance of dynamic 18F-FET PET in newly diagnosed astrocytic high-grade Glioma. J Nucl Med. 2015;56:9-15.

26. Thon N, Kunz M, Lemke L, Jansen NL, Eigenbrod S, Kreth S, et al. Dynamic 18F-FET PET in suspected WHO grade II gliomas defines distinct biological subgroups with different clinical courses. Int J Cancer J Int Du Cancer. 2015;136:2132-45.

27. Suchorska B, Giese A, Biczok A, Unterrainer M, Weller M, Drexler $\mathrm{M}$, et al. Identification of time-to-peak on dynamic 18F-FET-PET as a prognostic marker specifically in IDH1/2 mutant diffuse astrocytoma. Neuro-Oncology. 2018;20:279-88.

28. Hamacher K, Coenen HH. Efficient routine production of the $18 \mathrm{~F}$ labelled amino acid O-2-18F fluoroethyl-L-tyrosine. Appl Radiat Isot. 2002;57:853-6.

29. Langen KJ, Bartenstein P, Boecker H, Brust P, Coenen HH, Drzezga A, et al. German guidelines for brain tumour imaging by PET and SPECT using labelled amino acids. Nuklearmedizin. 2011;50:167-73.

30. Law I, Albert NL, Arbizu J, Boellaard R, Drzezga A, Galldiks N, et al. Joint EANM/EANO/RANO practice guidelines/SNMMI procedure standards for imaging of gliomas using PET with radiolabelled amino acids and [(18)F]FDG: version 1.0. Eur J Nucl Med Mol Imaging. 2019;46:540-57.

31. Herzog H, Langen KJ, Weirich C, Rota Kops E, Kaffanke J, Tellmann L, et al. High resolution BrainPET combined with simultaneous MRI. Nuklearmedizin. 2011;50:74-82.

32. Lohmann P, Herzog H, Rota Kops E, Stoffels G, Judov N, Filss C, et al. Dual-time-point O-(2-[(18)F]fluoroethyl)-L-tyrosine PET for grading of cerebral gliomas. Eur Radiol. 2015;25:3017-24.

33. Galldiks N, Stoffels G, Filss C, Rapp M, Blau T, Tscherpel C, et al. The use of dynamic O-(2-18F-fluoroethyl)-L-tyrosine PET in the diagnosis of patients with progressive and recurrent glioma. Neuro-Oncology. 2015;17:1293-300.

34. Rapp M, Heinzel A, Galldiks N, Stoffels G, Felsberg J, Ewelt C, et al. Diagnostic performance of 18F-FET PET in newly diagnosed cerebral lesions suggestive of glioma. J Nucl Med. 2013;54:229 35.

35. Pauleit D, Floeth F, Hamacher K, Riemenschneider MJ, Reifenberger G, Müller HW, et al. O-(2-[18F]fluoroethyl)-L-tyrosine PET combined with MRI improves the diagnostic assessment of cerebral gliomas. Brain. 2005;128:678-87.

36. Capper D, Zentgraf H, Balss J, Hartmann C, von Deimling A. Monoclonal antibody specific for IDH1 R132H mutation. Acta Neuropathol. 2009;118:599-601.

37. Capper D, Weissert S, Balss J, Habel A, Meyer J, Jager D, et al. Characterization of R132H mutation-specific IDH1 antibody binding in brain tumors. Brain Pathol. 2010;20:245-54.

38. Felsberg J, Rapp M, Loeser S, Fimmers R, Stummer W, Goeppert $\mathrm{M}$, et al. Prognostic significance of molecular markers and extent of 
resection in primary glioblastoma patients. Clin Cancer Res. 2009;15:6683-93.

39. Yokogami K, Yamasaki K, Matsumoto F, Yamashita S, Saito K, Tacheva A, et al. Impact of PCR-based molecular analysis in daily diagnosis for the patient with gliomas. Brain Tumor Pathol. 2018;35:141-7.

40. Wen PY, Macdonald DR, Reardon DA, Cloughesy TF, Sorensen AG, Galanis E, et al. Updated response assessment criteria for highgrade gliomas: response assessment in neuro-oncology working group. J Clin Oncol. 2010;28:1963-72.

41. Stupp R, Mason WP, van den Bent MJ, Weller M, Fisher B, Taphoorn MJ, et al. Radiotherapy plus concomitant and adjuvant temozolomide for glioblastoma. N Engl J Med. 2005;352:987-96.

42. Chinot OL, Wick W, Mason W, Henriksson R, Saran F, Nishikawa $\mathrm{R}$, et al. Bevacizumab plus radiotherapy-temozolomide for newly diagnosed glioblastoma. N Engl J Med. 2014;370:709-22.

43. Weller M, Butowski N, Tran DD, Recht LD, Lim M, Hirte H, et al. Rindopepimut with temozolomide for patients with newly diagnosed, EGFRvIII-expressing glioblastoma (ACT IV): a randomised, double-blind, international phase 3 trial. Lancet Oncol. 2017;18:1373-85.

44. Stupp R, Taillibert S, Kanner A, Read W, Steinberg DM, Lhermitte $B$, et al. Effect of tumor-treating fields plus maintenance temozolomide vs maintenance temozolomide alone on survival in patients with glioblastoma: a randomized clinical trial. Jama. 2017;318:2306-16.

45. Filss CP, Albert NL, Böning G, Kops ER, Suchorska B, Stoffels G, et al. O-(2-[(18)F]fluoroethyl)-L-tyrosine PET in gliomas: influence of data processing in different centres. EJNMMI Res. 2017;7:64.

46. Watanabe T, Nobusawa S, Kleihues P, Ohgaki H. IDH1 mutations are early events in the development of astrocytomas and oligodendrogliomas. Am J Pathol. 2009;174:1149-53.

47. Grasbon-Frodl EM, Kreth FW, Ruiter M, Schnell O, Bise K, Felsberg J, et al. Intratumoral homogeneity of MGMT promoter hypermethylation as demonstrated in serial stereotactic specimens from anaplastic astrocytomas and glioblastomas. Int J Cancer J Int Du Cancer. 2007;121:2458-64.

48. Thon N, Eigenbrod S, Grasbon-Frodl EM, Ruiter M, Mehrkens JH, Kreth S, et al. Novel molecular stereotactic biopsy procedures reveal intratumoral homogeneity of loss of heterozygosity of $1 \mathrm{p} / 19 \mathrm{q}$ and TP53 mutations in World Health Organization grade II gliomas. J Neuropathol Exp Neurol. 2009;68:1219-28.

Publisher's note Springer Nature remains neutral with regard to jurisdictional claims in published maps and institutional affiliations. 